\title{
Value Analysis of Fetal System Ultrasound Examination and Routine Obstetric Ultrasound Examination
}

\author{
Lianjie Bai ${ }^{1}$ and Huilin Liu ${ }^{2}$ \\ ${ }^{12}$ The Second Affiliated Hospital of Qigihar Medical University,Department of Ultrasonic Teaching \\ and Research, Qigihar 161000, Heilongjiang,China \\ a bljdxx@163.com
} Keywords: Fetal system ultrasound examination; Routine obstetric Ultrasound examination;
Diagnosis

\begin{abstract}
Objective: The two methods of fetal system ultrasound examination and routine obstetric ultrasound examination were studied, and its application value was analyzed. Method: In this period from May 2016 to May 2017, 120 patients in the late pregnancy were examined in our hospital as the subject of this study. 60 of them were performed routine obstetric ultrasound examination as group A, the other 60 patients developed fetal system ultrasound examination, for group B, the diagnosis of two methods of examination was analyzed. Result: Clinical pathology confirmed that there were 11 malformed fetuses in group A and 10 cases of malformed fetus in group B, of which 6 cases $(54.5 \%)$ were diagnosed by routine obstetric ultrasound examination in group A, and 9 cases $(90 \%)$ of malformed fetus were diagnosed by fetal system ultrasound in group B, and the diagnostic accuracy of B group was higher than that of group A, the difference was obvious. Conclusion: Through comparative analysis, fetal system ultrasound examination for the diagnosis of malformed fetus is more accurate than routine obstetric ultrasound examination.

Due to changes in environmental conditions and lifestyles, in recent years, the number of newborns in our country has been increasing year by year. At the same time, the incidence of malformed fetuses has gradually increased, which has reduced the quality of our population. In the clinic, routine obstetric ultrasound examination has been unable to meet, its operation is very simple, but the need to use the examination time is relatively short, for abnormal conditions of the fetus is not easy to reach the effect of detailed diagnosis. With rising of diagnosis technology, it is gradually applied to the fetal system ultrasound examination. It can mainly carry out a series of examinations for specific fetal problems and belongs to a class of three-dimensional color doppler ultrasound examinations ${ }^{[1-2]}$. It can be used in the diagnosis of fetal hydrocephalus, microcephaly and heart malformation. It can be used in clinical examination, it is beneficial to discover problems in time and take treatment measures, which is beneficial to reduce the birth rate of malformed newborns and has important application value. The main purpose of this study is to explore the diagnosis of fetal system ultrasound examination and routine obstetric ultrasound examination. The present report is as follows.
\end{abstract}

\section{Information and Methods}

\section{General Information}

In this period from May 2016 to May 2017, 120 patients in the late pregnancy were examined in our hospital as the subject of this study. 60 of them were performed routine obstetric ultrasound examination as group $\mathrm{A}$, the other 60 patients developed fetal system ultrasound examination, for group B. Group A: the age of pregnant women is between 24 and 38 years old, the median age is $(28.1 \pm 1.5)$ years, and the number of pregnant weeks is mostly 23 weeks to 26 weeks; Group B: the age of pregnant women is between 23 and 38 years old, the median age is (27.5 \pm 1.8$)$ years, and the number of pregnant weeks is mostly 24 weeks to 27 weeks. There was no significant difference between the two groups in statistics analysis.

\section{Method}

(1) Group A: routine prenatal ultrasound examination, mainly select the location of the fetus, 
placenta position, fetal growth and other examination, and then the cervix and placenta to explore, check fetal head circumference, abdominal circumference, weight, and so on ${ }^{[3]}$, the examination time for 5 minutes.

(2) Group B: fetal system ultrasound examination, including the fetus's neck, face, abdomen, placenta, spine, and so on, and an accurate examination of the abdomen of a pregnant woman is also carried out. The examination of the project has bone and internal structure, and the internal unfolding of different sections is examined. For example, when examining the fetal head, three different sections of the cerebellum, thalamus, and lateral ventricle need to be included; For thoracic cavity examination, we need to observe the relationship between quadruple structure of the heart and understand the lung morphology. At the same time, we need to check the abdomen of pregnant women, understand the shape of the umbilical cord outlet position, observe the umbilical cord around the drum and around the neck.

\section{Evaluation index ${ }^{[4-5]}$}

In this study, two groups of pregnant women were confirmed by clinical pathology. There were 11 cases of malformed fetus in group A, and 10 cases of malformed fetus in group B. Therefore, the evaluation index is two cases of malformed fetus in pregnant women, and the two methods are compared.

\section{Statistical method}

In the statistics of the medical statistics software SPSS 17 on the obtained data, the data are expressed with\% of the count data and detected by $\mathrm{x}^{2}$; the measurement data is expressed in $(\bar{x} \pm s)$ and $\mathrm{T}$, and if the numerical $\mathrm{P}<0.05$ is obtained, there is a significant difference between the data and the statistical significance.

\section{Result}

Two different examination methods were used to examine the pregnant women selected in this study. There were 11 cases of malformed fetus in group A and 10 cases of malformed fetus in group $\mathrm{B}$, and 6 cases of malformed fetus were diagnosed by routine obstetric ultrasound examination in group A, the accuracy rate was $54.5 \%$, the fetus was 2 cases of thoracic and ascites, 2 cases of neural tube defects, 1 cases of cleft lip and palate and 1 cases of congenital heart disease; in group B, 9 cases of malformed fetus were diagnosed by fetal system ultrasound examination, and the accuracy rate was $90 \%$. Among them, there were 3 cases of thoracic and ascites, 4 cases of neural tube malformation, 1 cases of cleft lip and palate and 1 cases of congenital heart disease. By contrast, the accuracy of diagnosis in group B was higher than that in group A, and the difference was obvious by statistical analysis, $\mathrm{x}^{2}=30.556, \mathrm{p}=0.000$.

\section{Discussion}

Our country belongs to a large population, the number of newborn babies is up to about twenty million every year, but the number of malformed newborns in these newborns is $1.5 \%{ }^{[6]}$. Some of them are partly due to the lack of awareness of the obstetric ultrasound examination. Therefore, in order to prevent the number of malformed newborns increasing and promoting the quality of the population in our country, the obstetric ultrasound examination should be strengthened. It is beneficial to reduce the birth rate of malformed newborns by finding problems in time and taking measures to deal with them. In the clinic, more examination methods for pregnant women in the late pregnancy are mainly routine prenatal examination, which is very simple in operation. However, the time of examination is short, and it is not easy to achieve the effect of detailed diagnosis for malformed fetal situation, and with the continuous improvement of clinical diagnosis technology, fetal system ultrasound examination is gradually adopted ${ }^{[7-8]}$. It is mainly aimed at the examination of fetal problems ${ }^{[9-10]}$. It belongs to a class of three-dimensional color doppler ultrasound examination, which includes ultrasound examination and targeted ultrasound examination of the middle and late pregnancy fetus system. It can accurately diagnose the fetal hydrocephalus, microcephaly, Down syndrome and so on, so it has been widely used in clinical. Compared with 
routine obstetric ultrasound examination, fetal system ultrasound examination has the advantages of convenient operation, high safety and small wound. It provides a comprehensive and comprehensive clinical analysis of the maternal women who are in the middle and late stages of pregnancy. It is clear whether the fetus has abnormal condition, whether there is hydrocephalus and congenital heart disease. In this study, the routine obstetric ultrasound examination and fetal system ultrasound examination were carried out for the late pregnant women in our hospital. The data showed that the accuracy of fetal system ultrasound examination was higher than that of routine obstetric ultrasound examination, and there was a more obvious difference.

But in the process of examination, it is found that fetal system ultrasound examination is easily influenced by the position of fetus, pregnancy and other factors, and has a certain influence on the accuracy of diagnosis. Therefore, the examination time must be controlled well for the examination of pregnant women. The first examination should be chosen about 23 weeks. In this period, the fetus is basically mature and has a clear fetal movement. The examination of ultrasound imaging can clearly show the specific status of the fetus in the maternal body. The second examination can be selected in 31 weeks. The fetal heart and four cavity structures are in the stage of gradual maturity, and the heart of the fetus can be clearly displayed. Therefore, fetal system ultrasound examination in middle and late pregnancy has the highest diagnostic rate. However, there are some limitations in the application of the diagnostic method to the clinic at present. It is mainly due to the high requirements and high cost of the equipment needed in the inspection process, and it requires the doctor to have excellent operation technology. But as a whole, the method has a high value of application. The malformed fetus can be found in time, so as to carry out the targeted measures. Therefore, this method can be applied in clinical application.

\section{Reference}

[1] F Conti, J Park. Interface Design and Control Strategies for a Robot Assisted Ultrasonic Examination System [J]. Springer Berlin Heidelberg, 2014, 79 :97-113

[2] M Tabatabaeipour. Non-destructive ultrasonic examination of root defects in friction stir welded butt-joints [J]. Ndt \& E International, 2016, 80 :23-34

[3] X Wang, D Liu. Using critical care chest ultrasonic examination in emergency consultation: a pilot study [J]. Ultrasound in Medicine \& Biology, 2015, 41 (2) :401-406

[4] M Wu, G Shao. Estimation of fetal weight by ultrasonic examination [J]. Int J Clin Exp Med, 2015, 8 (1): :540-545

[5] H Alizadeh, $\mathrm{N}$ Najmi. Diagnostic accuracy of ultrasonic examination in suspected craniosynostosis among infants [J]. Indian Pediatrics, 2012, 50 (1) :148-150

[6] HU Qiu-Yun, CP Chen. Prenatal systematic ultrasonic examination in diagnosis of fetal congenital malformations [J]. Chinese Journal of Medical Imaging Technology, 2012, 28 (2) :343-346

[7] H Qiu, BU Room. Clinical Value of B Ultrasonic Examination for Ureteral Calculi [J]. Clinical Medicine \& Engineering, 2014

[8] Y Ding, L Sun. Cervical changes during second trimester by ultrasonic examination [J]. Journal of Hainan Medical University, 2013

[9] Wang Hong-ling, Li Feng. Clinical observation of ultrasonic examination in the diagnosis of late pregnancy and obstetric hemorrhage [J]. World Latest Medicine Information, 2016

[10] BJ Kimura. Cardiopulmonary Limited Ultrasound Examination for "Quick-Look" Bedside Application [J]. American Journal of Cardiology, 2013, 108 (4) :586-590 